\title{
Quantum-chromodynamic phenomenology of gluon jets
}

\author{
K. Shizuya \\ Fermi National Accelerator Laboratory, Batavia, Illinois 60510* \\ S.-H. H. Tye \\ Laboratory of Nuclear Studies, Cornell University, Ithaca, New York 14853
}

(Received 20 February 1979)

\begin{abstract}
We investigate in detail, within the framework of perturbative quantum chromodynamics, properties of the gluon jet in comparison with those of the quark jet. The short-distance features of a gluon jet are quite similar to those of a quark jet, apart from the difference in the overall color charges $C_{2}(G) / C_{2}(R)=9 / 4$. The spread of a gluon jet in the transverse momentum is much bigger than that of a quark jet and the narrowing of a gluon jet with increasing energy is slower than that of a quark jet. We also evaluate the thrust $T$ distribution which has the same features for both types of hadronic jets. The average $\langle 1-T\rangle$ and higher "thrust moments" $\left\langle(1-T)^{n}\right\rangle$ for the gluon-jet case are again approximately $C_{2}(G) / C_{2}(R)=9 / 4$ times those for the quark-jet case. The usefulness of these thrust moments is emphasized. The implication of the above result on the search for gluon jets is discussed, especially in the hadronic decay of a heavy quarkonium state. We express the gluon-jet production cross section in terms of the Altarelli-Parisi parton functions. We emphasize that the spread of the gluon jet is a very clean place to measure the triple-gluon vertex coupling, since it provides the dominant contribution in the transversemomentum spread.
\end{abstract}

\section{INTRODUCTION}

It appears likely that strong interactions are governed by the non-Abelian gauge-field theoryquantum chromodynamics (QCD) - which involves elementary quark and gluon fields. The evidence for the existence of spin- $-\frac{1}{2}$ quarks is overwhelming. All evidences for the existence of colored gluons, on the other hand, are circumstantial at best. The effects of gluonic degrees of freedom have so far been seen in the form of deviations from the naive quark-parton picture. Therefore, to put QCD on firmer grounds, it is essential to search for more direct signatures of the existence of vector gluons.

It is generally believed that, owing to some nonperturbative color-confinement effects in QCD, quarks and gluons cannot be seen by themselves but only in the form of hadrons which are color singlets. Therefore, one must search for some other manifestations (as direct as possible) of the fundamental quanta. The hadronic-jet phenomena observed in $e^{+} e^{-}$annihilation ${ }^{1}$ and other experiments are considered to be induced by energetic quarks which fragment into hadrons. Similarly, it is expected that an energetic gluon would also manifest itself in the form of a hadronic jet. In lieu of a free gluon, gluon jets (i.e., hadronic jets arising from energetic gluons) should best reflect the features of the colored vector quanta. In this work we continue our study ${ }^{2}$ on the properties of gluon jets in comparison with those of quark jets, within the framework of perturbative QCD.
The quark-confinement effect in QCD is not yet understood. With the idea of asymptotic freedom, ${ }^{3}$ QCD perturbation theory is reliable only for the calculations of short-distance effects. Correspondingly, to calculate any quantitative properties of quarks and gluons by means of QCD perturbation theory, one must take great care to isolate perturbative short-distance effects from nonperturbative quark-confinement effects. In the case of deep-inelastic lepton-hadron scattering, this type of isolation of short-distance effects is achieved by use of Wilson's operator-product expansion along with the renormalization group. ${ }^{3}$ There are also attempts ${ }^{4,5}$ to extend the application of QCD perturbation theory to other processes where the analysis based on the operator-product expansion and the renormalization group is not directly applicable.

Recently, many authors, in particular Politzer ${ }^{4}$ and Sterman and Weinberg, ${ }^{5}$ have suggested that suitably chosen features of hadronic final states produced in scattering processes may be calculable by means of QCD perturbation theory, provided (1) nonperturbative confinement effects are either factorized or smeared over and (2) all infrared divergences and mass singularities are absent.

Following this criterion, Sterman and Weinberg have studied, using perturbative QCD, the hadronic-jet structure observed in $e^{+} e^{-}$annihilation. They define the cross section in terms of jetlike hadronic final states, characterized by the backto-back cone of half-angle $\delta$, in which all but a small fraction $\epsilon$ of the total $e^{+} e^{-}$energy $E$ is emitted. Figure 1(a) shows the basic process that 


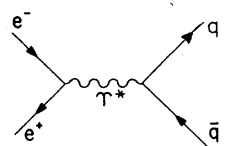

(a)

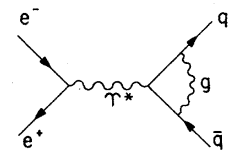

(c)

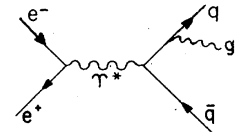

(b)

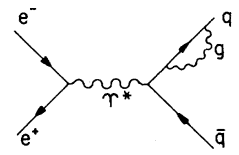

(d)
FIG. 1. $e^{+} e^{-}$annihilation processes that lead to hadronic-jet phenomena. The wavy line denoted by $\gamma^{*}$ is the virtual photon. $q$ and $g$ stand for a quark and a gluon, respectively.

leads to quark jets. A pair of energetic quark and antiquark produced via the virtual-photon decay into hadrons due to some nonperturbative long-range effects in QCD. The gluon bremsstrahlung process [Fig. 1(b)] leads to the broadening of hadronic jets (which we shall call quark jets). The fraction of the jetlike events characterized by $\epsilon$ and $\delta$ in the total events is, to leading order in $\alpha_{C}=g^{2} / 4 \pi$, given by ${ }^{5}$

$$
f(\text { quark })=1-\frac{\alpha_{C}}{\pi} C_{2}(R)\left[(4 \ln 2 \epsilon+3) \ln \delta+\frac{\pi^{2}}{3}-\frac{7}{4}\right],
$$

where terms of order $\epsilon$ and/or $\delta$ are neglected and the color factor $C_{2}(R)=\frac{4}{3}$ for triplet quarks. This expression is free of infrared divergences and mass singularities since they are canceled between the real-particle-emission process [Fig. $1(b)$ ] and the virtual correction processes [Figs. $1(c)$ and $1(d)]$. In a sense, the long-range effects are smeared over the energy resolution $\epsilon$ and the angular radius $\delta$ so that only the short-distance features of jet phenomena survive. The ratio $f$ provides a measure of how jetlike is the quark jet.

This approach is particularly useful for the study of gluon jets for the following reasons: (1) The perturbative QCD calculation of the fraction $f$ for the gluon-jet case is also free of infrared and mass singularities. (2) The property calculated is qualitatively independent of the source of the energetic gluon produced; hence we are led to believe that it is applicable to many scattering processes where the production of gluon jets is expected: for example, lepton-hadron deep-inelastic scattering, Drell-Yan dilepton production, large $-p_{\perp}$ scattering experiments, $e^{+} e^{-}$annihilation into hadrons, and the hadronic decays of heavy-quark bound states. (3). The calculation does not require any knowledge of how an ener- getic gluon fragments into hadrons, as long as the overall transverse-momentum spread of the gluon-jet fragmentation does not differ drastically from that of the quark-jet fragmentation. This last point is of particular importance since we know nothing about the gluon fragmentation function.

Using this approach, we have shown that qualitatively a gluon jet tends to spread substantially more than a quark jet. ${ }^{2,6,7}$ of course, this conclusion is applicable only to kinematic regions where QCD perturbation theory is valid while nonperturbative effects are small. Unfortunately, at energies available today or in the near future the kinematic region where such a quantitative comparison is valid is rather limited. Hence it is worthwhile to use a more suitable variable instead of $f(\epsilon, \delta)$ for a quantitative comparison between the quark jet and the gluon jet. In this work we choose the variable thrust ${ }^{8} T, \frac{1}{2} \leqslant T \leqslant 1$,

$$
T=\max \left(\sum_{i}\left|\overrightarrow{\mathrm{p}}_{\| i}\right| / \sum_{i}\left|\overrightarrow{\mathrm{p}}_{i}\right|\right),
$$

where the sum runs over all observed particles and the $\vec{p}_{\|}$are parallel to a "jet" axis which is chosen to maximize $T$. This axis is called the thrust axis. (Our definition differs slightly from that proposed originally by $\mathrm{Farhi}^{8}$; we believe this definition is better in practice since, for an event where some hadrons escape detection, the thrust $T$ in his definition may attain values beyond the bound $\frac{1}{2} \leqslant T \leqslant 1$. The two definitions coincide for a perfect detector.) In terms of the thrust distribution, we find that the gluon jet is again less jetlike than the quark jet. This means that the observation of a gluon jet is difficult to achieve.

In perturbative QCD the transverse-momentum spread of a gluon jet arises from gluon-pair or quark-pair emission. At high energies the structure of those particle emission processes is determined by that of mass singularities associated with them. It was noticed by a number of authors ${ }^{4,7}$ that the leading mass singularities in QCD have a structure universal to many high-energy processes. In physically sensible quantities such as the inclusive jet cross section, those mass singularities as well as infrared divergences are expected to be canceled ${ }^{9}$ between the real and virtual correction processes, leaving their shortdistance features which reflect the structure of the mass singularities. This in turn implies that the high-energy features of a gluon jet are independent of how it is produced. It will be reasonable to suppose that the production of energetic gluons is insensitive to the nonperturbative confinement effect which induces a gluon to fragment 
into hadrons; this is because the energetic-gluon production is considered to take place in a much shorter time scale than the latter.

With this in mind we can study the gluon-jet property in perturbative QCD by choosing the simplest, well-understood operator as a gluon production source. The hadronic jet production has a clear parton interpretation. To emphasize the parton language, we discuss the scalar source and the pseudoscalar source in two different presentations. We shall express the inclusive jet cross section in terms of the Altarelli-Parisi parton functions ${ }^{10}$ in the Appendix.

Quantitatively, the cross section $(1 / \sigma)(d \sigma / d T)$ for either gluon-pair or quark-pair production is peaked at $T \rightarrow 1$. This is the case even after all infrared and mass singularities are canceled between the real-and virtual-particle-emission diagrams. Physically, events with smaller $p_{\perp}$ (or equivalently $T \rightarrow 1$ ) would be crowded with particles arising from confinement effects, as chracterized by a limited average transverse momentum $\left\langle p_{\perp}\right\rangle \sim 0.3 \mathrm{GeV}$. Even within perturbative QCD, the Kinoshita-Lee-Nauenberg theorem ${ }^{9}$ requires us to average the cross section over the detection resolution size $\Delta T$ of $T$ to remove the small-momentum singularities at $T \rightarrow 1$. Therefore, to render the perturbative calculation sensible, we must average the cross section over a resolution $\Delta T$ larger than the uncertainty $\Delta T$ (nonperturbative) induced by the fragmentation process. ${ }^{11}$ For example, we can use the inclusive cross section

$$
\sigma(\Delta T)=\int_{1-\Delta T}^{1} d T(d \sigma / d t)
$$

with $\Delta T>\Delta T$ (nonperturbative).

As an alternative to the above smearing procedure, we suggest using some "large- $T$ suppressed" expression for the comparison between theory and experiment. Let us introduce $(1-T)^{n}\left(1 / \sigma_{\text {tot }}\right)(d \sigma / d T)$ for $n \geqslant 0$. For positive integer $n$, the small $-T$ region (where perturbative QCD effects dominate) is enhanced relative to the large- $T$ region (where infrared and confinement effects are large). In particular, for the gluonpair and quark-pair production discussed in Sec. III, we can use $(1-T)\left(1 / \sigma_{\text {tot }}\right)(d \sigma / d T)$ so that the leading infrared and mass singularities in $d \sigma / d T$ [which are proportional to $\delta(1-T)$ ] are suppressed. It will be equally useful to consider the integrated quantities

$$
\begin{aligned}
M_{n}(E) & =\left\langle(1-T)^{n}\right\rangle \\
& =\int_{1 / 2}^{1} d T(1-T)^{n}\left(1 / \sigma_{\text {tot }}\right)(d \sigma / d T),
\end{aligned}
$$

which we shall refer to as thrust moments at energy $E$. It is clear that to leading order in perturbative $\mathrm{QCD}$, all thrust moments are proportional to the effective running coupling constant $\alpha_{C}(E)$.

The rest of this paper is organized as follows: In Sec. II, we present the doubly differential cross section for the production of a pair of gluon jets from a color-singlet gauge-invariant scalar source $\left(F_{\mu \nu}^{a}\right)^{2}$, in leading-order perturbative QCD, as a function of the energies of the quanta. We evaluate the total cross section which includes both virtual and real emissions. In Sec. III, we derive the fraction $f(\epsilon, \delta ; E)$ with the inclusion of the constant term in it. We also derive the differential cross section expressed in terms of the thrust and compare the gluon-jet and quark-jet productions. Nonperturbative effects are also discussed. Section IV contains discussions and remarks on how best to find gluon jets, in view of the prediction that they are broader in transverse momentum than quark jets. In the Appendix we show that the pseudoscalar source $\left(F_{\mu \nu}^{a} \tilde{F}_{\mu \nu}^{a}\right)$ gives the same gluon-jet structure as the scalar source.

\section{GLUON-JET PRODUCTION CROSS SECTION}

To study the gluon-jet properties by means of QCD perturbation theory, we consider the gluonjet production from the color-singlet, gaugeinvariant, scalar source $F_{\mu \nu}^{a}[A] F_{\mu \nu}^{a}[A]$, where

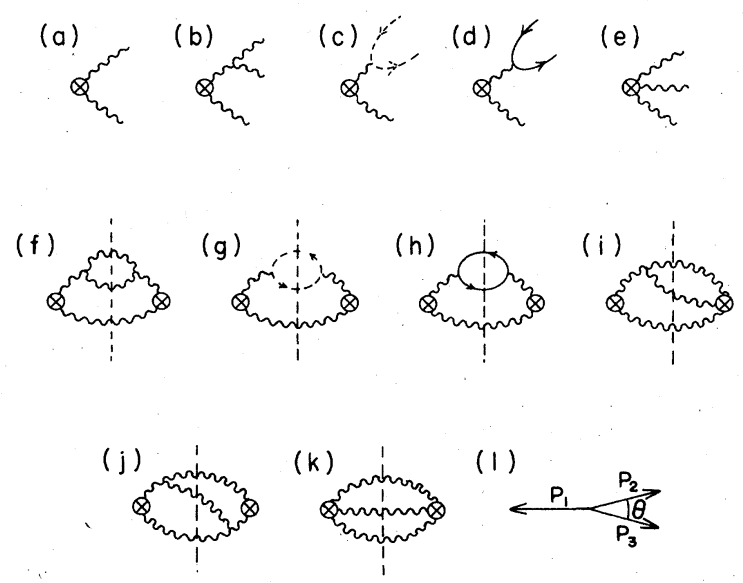

FIG. 2. Gluon-jet production from a source. Wavy lines are gluons, dotted lines are Faddeev-Popov ghosts, and solid lines are massless quarks. (a) lowest-order diagram. (b) - (e) particle-emission processes to leading order. (f) - (k) squared amplitudes; the dashed lines denote unitary cuts. Mirror reflections of these diagrams are not shown. (1) three-body configuration. 


$$
F_{\mu \nu}^{a}[A]=\partial_{\mu} A_{\nu}^{a}-\partial_{\nu} A_{\mu}^{a}+g f^{a b c} A_{\mu}^{b} A_{\nu}^{c} .
$$

To zeroth order in the coupling constant $g$, a pair of gluons is produced from this source. To investigate the short-distance effects on the gluonjet spreading we calculate the gluon-jet production cross section in the one-loop approximation, which subsequently is promoted, on the basis of the argument of Sterman and Weinberg, ${ }^{5}$ to the leading-logarithmic approximation. In this section we evaluate the differential cross section and the total cross section to order $\alpha_{C}=g^{2} / 4 \pi$.

Figure 2(a) shows the basic process that leads to the production of a pair of gluon jets. Figures 2(b) -2 (e) are particle-emission processes to order $g$, where the final states have either three gluons or a gluon plus a quark pair. All quark masses are taken to be zero. We shall perform our calculation in the Feynman gauge, and correspondingly we have to include the fictitious final state of a gluon plus a ghost pair [Fig. 2(c)] (we can of course avoid the ghost-pair production diagram by working in the axial gauge). In order to regularize the infrared divergences and mass singularities we shall work in $n=4+D$ dimensions. ${ }^{12}$ The three-body Lorentz-invariant phase space is fivefold differential (in four dimensions). Since the angular distribution of the gluon production cross section depends on the source and is of no interest to us, we shall integrate over all angles, leaving only two independent energy fractions $x_{i}=2 E_{i} / E(i=1,2,3), x_{1}+x_{2}+x_{3}=2$, and 0 $\leqslant x_{i} \leqslant 1\left(E_{i}\right.$ is the energy carried by the massless particle $i$ and $E$ is the center-of-mass total energy).

A straightforward calculation yields the following doubly differential cross section for the sum of real-particle-emission processes [Figs. 2(f)$2(\mathrm{k})]$ in $n=4+D$ dimensions:

$$
\begin{aligned}
\frac{1}{\sigma_{0}} \frac{d \sigma(1)}{d x_{1} d x_{2}}=\frac{\alpha_{C}}{3 \pi} f(D) E^{D}\{ & C_{2}(G)\left[\frac{11}{6}-\frac{5}{2} \frac{1}{1-x_{1}}+\frac{\left(1-x_{2}\right)\left(1-x_{3}\right)}{1-x_{1}}+\frac{1}{2} \frac{\left(1-x_{2}\right)^{2}+\left(1-x_{3}\right)^{2}+2}{\left(1-x_{2}\right)\left(1-x_{3}\right)}\right] \\
& \left.+T(R) N_{f} \frac{1}{\left(1-x_{1}\right)}\left[1-(2-D)\left(1-x_{2}\right)\left(1-x_{3}\right)\right]+\text { symmetrization in }\left(x_{1}, x_{2}, x_{3}\right)\right\} .
\end{aligned}
$$

Here $\alpha_{C}=g^{2} /(4 \pi)$ is the QCD coupling constant and $\sigma_{0}$ is the total cross section for the Born diagram Fig. 2(a): $f(D) \equiv\left[(2 \sqrt{\pi})^{D} \Gamma\left(1+\frac{1}{2} D\right)\right]^{-1}$, where $f(D)=1$ for $D=0 . C_{2}(G)$ is the value of the quadratic Casimir operator for the color representation $G$ of the gluon; in QCD $C_{2}(G)=C_{2}(8)=3 . T(R)$ is given by the trace of the product of two colorrepresentation matrices of the quark; for triplet quarks $T(R)=\frac{1}{2}$. $N_{f}$ is the number of quark flavors. The symmetrization means $\left(x_{1} \rightarrow x_{2} \rightarrow x_{3} \rightarrow x_{1}\right)$ and $\left(x_{1}-x_{3}-x_{2}-x_{1}\right)$, so that the sum of the constant terms is three times of that shown in (2.1). For later convenience we have also fully symmetrized the quark-pair-emission contribution, which originally is symmetric in the quark and antiquark energy fractions but not in the gluon energy fraction. We observe that diagram Fig. 2(e) contributes only a constant term.

It is a simple exercise to derive the three-body phase space in terms of $x_{i}$ in $n=4+D$ dimensions ${ }^{13}$ :

$$
\begin{aligned}
d R= & d x_{1} d x_{2} d x_{3} \delta\left(2-x_{1}-x_{2}-x_{3}\right) \\
& \times\left[\left(1-x_{1}\right)\left(1-x_{2}\right)\left(1-x_{3}\right)\right]^{D / 2} .
\end{aligned}
$$

The physical region is given by the Dalitz triangle region shown in Fig. 3. The three sides $\left(x_{i}=1\right)$ of the triangle correspond to two-jet-like configurations where two of the three final-state particles are collinear. The differential cross section (2.1) is singular for $x_{i}=1$; this gives rise to mass singularities. The three corners of the triangle, where one of $x_{i}$ is close to zero, correspond to soft-particle emissions and lead to infrared divergences as well as mass singularities for the soft-gluon-emission processes [Figs.

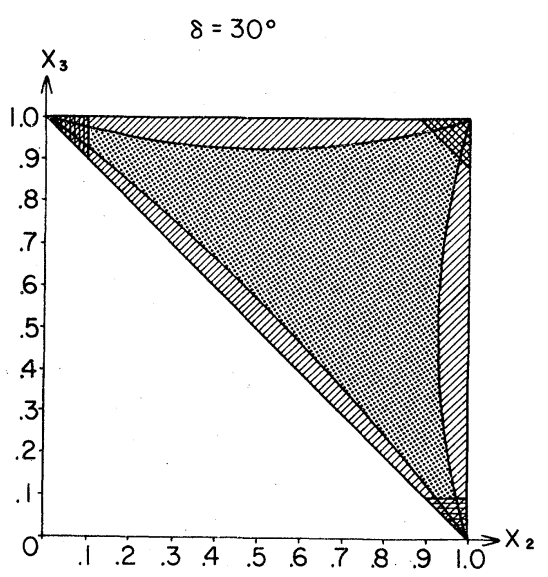

FIG. 3. Three-body phase space expressed in terms of the energy fractions $x_{i}(i=1,2,3)$ (Dalitz plot). The physical region is $0 \leqslant x_{i} \leqslant 1, x_{1}+x_{2}+x_{3}=2$. The shaded area along the boundary of the phase-space region corresponds to the two-jetlike final-state configuration specified by $\epsilon$ and $\delta$. 
$2(\mathrm{f}), 2(\mathrm{~g}), 2(\mathrm{i})$, and $2(\mathrm{j})]$. The quark-pair-emission process [Fig. 2(h)] has only a mass singularity.

The integration of the differential cross section
(2.1) over the whole phase space gives the cross section $\sigma(1)$ for the real-particle-emission processes:

$$
\begin{aligned}
\sigma(1) & =\int_{0}^{1} d x_{1} \int_{1-x_{1}}^{1} d x_{2}\left[\left(1-x_{1}\right)\left(1-x_{2}\right)\left(1-x_{3}\right)\right]^{D / 2}\left[d \sigma(1) / d x_{1} d x_{2}\right] \\
& =\sigma_{0} \frac{\alpha_{C}}{\pi} f(D) E^{D}\left[C_{2}(G)\left(\frac{4}{D^{2}}-\frac{11}{3} \frac{1}{D}+\frac{73}{12}-\frac{\pi^{2}}{2}\right)+N_{f} T(R)\left(\frac{4}{3} \frac{1}{D}-\frac{7}{3}\right)\right] .
\end{aligned}
$$

The single poles in $D=n-4$ represent mass singularities while the double pole arises from the coalescence of a mass singularity and an infrared divergence at the corners of the Dalitz triangle.

These divergences are canceled ${ }^{9}$ by those arising from the virtual corrections to the same order in $\alpha_{C}$, which are shown in Fig. 4. The total cross section $\sigma(2)$ for the virtual correction processes to order $\alpha_{C}$ has previously been calculated ${ }^{2}$ :

$$
\sigma(2)=\sigma_{0} \frac{\alpha_{C}}{\pi} f(D)\left[C_{2}(G)\left(-\frac{4}{D^{2}} E^{D}+\frac{11}{3} \frac{\mu^{D}}{D}-\frac{67}{18}\right)+N_{f} T(R)\left(-\frac{4}{3} \frac{\mu^{D}}{D}+\frac{20}{9}\right)\right],
$$

where $\mu$ denotes the subtraction point $p^{2}=\mu^{2}$ at which we have performed the renormalization of the gluon propagators. For the renormalization of the scalar source $\left(F_{\mu \nu}[A]\right)^{2}$ we have subtracted only the ultraviolet-divergent piece at $p^{2}=\mu^{2}$ for gluon lines and at $p^{2}=E^{2}$ for the source momentum.

As a consequence of the cancellation of the infrared divergences and mass singularities between the real and virtual corrections, the total cross section to order $\alpha_{C}, \sigma_{\text {tot }}=\sigma_{0}+\sigma(1)+\sigma(2)$ is finite $(D \rightarrow 0)$ :

$$
\sigma_{\text {tot }}=\sigma_{0}\left\{1-\frac{\alpha_{C}}{\pi}\left[\left(\frac{11}{3} C_{2}(G)-\frac{4}{3} N_{f} T(R)\right) \ln (E / \mu)+\left(\frac{\pi^{2}}{2}-\frac{85}{36}\right) C_{2}(G)+\frac{1}{9} N_{f} T(R)\right]\right\} \text {. }
$$

The source we have used is not a conserved source. Accordingly, the coefficient of the $\ln (E / \mu)$ term in (2.5) represents the anomalous dimension ${ }^{14}$ of the scalar source $\left(F_{\mu \nu}[A]\right)^{2}$. It will be important to remark that the constant terms in the virtual correction (2.4) are somewhat ambiguous in the sense that they depend on how we renormalize the source (we have employed an off-mass-shell renormalization procedure). This is because one cannot use, due to the presence of infrared divergences and mass singularities, the on-massshell renormalization for exclusive processes such as the virtual correction (2.4). However, the physical quantity we are interested in, namely the fraction $f^{\prime}(\epsilon, \delta, E)$, is free of the ambiguity in $\sigma_{t \text { ot }}$; we shall discuss this point in the next section.

In general the operator $\left(F_{\mu \nu}[A]\right)^{2}$ is mixed, under renormalization, with the gauge-invariant operator $i \bar{q}^{a} \gamma^{\mu} D_{\mu}^{a b}[A] q^{b}$, where $D_{\mu}^{a b}[A]$ is the covariant derivative for the colored quark field $q^{b}(x)$. With the help of the equation of motion, this operator turns out to be proportional to the quark mass operator $\bar{q}^{a} m q^{a}$ which vanishes for massless quarks (i.e., a soft operator). Therefore, it is not necessary to consider the mixing with the quark operator. Since we calculate, physical quantities, it is also unnecessary to take explicit account of the mixing with non-gauge-invariant operators. ${ }^{14}$

\section{TRANSVERSE-MOMENTUM SPREAD OF GLUON JETS}

We are now ready to study the spread of the gluon jet due to short-distance QCD effects and compare it to that of the quark jet. We calculate the fraction $f(\epsilon, \delta, E)$ of two-jet-like events specified by $\epsilon$ and $\delta$ in the total events. We then calculate the thrust distribution from Eq. (2.1) and compare it to the quark-jet case. In the Appendix,

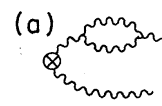

(e)
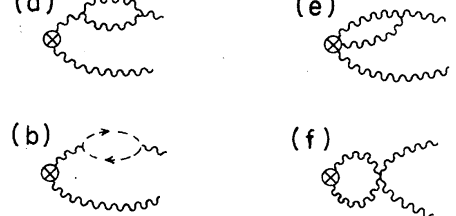

(f)
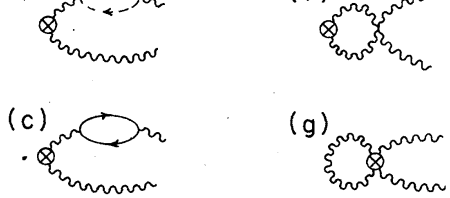

(d)

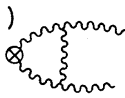

FIG. 4. Leading-order virtual corrections to twogluon production. Diagrams (f) and (g) involve only ultraviolet divergences. 
we present our previous method ${ }^{2}$ of calculation which is essentially the equivalent-photon approximation of Weizsäcker and Williams. ${ }^{15}$ This method was extensively used in the generalized parton language of Altarelli and Parisi. ${ }^{10}$

Let us first determine the kinematical regions which contribute to the inclusive cross section $\sigma(\epsilon, \delta)$ for jetlike final states. A jetlike configuration is characterized by a hadronic cone of half angle $\delta$ inside which all but a small fraction $\epsilon$ of the total energy $E$ is emitted. Suppose we consider the configuration where $x_{1}>x_{2}, x_{3}$ [see Fig. 2(1)]. The angle $\theta$ between $\overrightarrow{\mathrm{p}}_{2}$ and $\overrightarrow{\mathrm{p}}_{3}$ is given in terms of $x_{1}, x_{2}$, and $x_{3}$ by

$$
\sin ^{2}\left(\frac{1}{2} \theta\right)=\left(1-x_{1}\right) /\left(x_{2} x_{3}\right) \text {. }
$$

Therefore, $0 \leqslant \theta \leqslant 2 \delta$ when

$$
1-x_{1}=x_{2}+x_{3}-1 \leqslant\left(\sin ^{2} \delta\right) x_{2} x_{3},
$$

which is, together with the two other similar regions, shown as a shaded region in Fig. 3 . The hard-momentum regions are characterized by $2 \epsilon \leqslant x_{i} \leqslant 1-2 \epsilon(i=1,2,3)$ while the soft-momentum regions are the three remaining regions on the corners of the triangle.

The inclusive jet cross section is obtained by integrating the differential cross section (2.1) over the shaded area (hard + soft) in the Dalitz triangle. ${ }^{16}$ In particular, for the hard-momentum region near $x_{1} \simeq 1$, i.e., $x_{2}+x_{3}-1 \leqslant \delta^{2} x_{2} x_{3}$ and $2 \epsilon \leqslant x_{2} \leqslant 1-2 \epsilon$, the phase-space integration is written as

$$
\begin{aligned}
& \int_{2 \epsilon}^{1-2 \epsilon} d x_{2} \int_{1-x_{2}}^{\left(1-x_{2}\right) /\left(1-62 x_{2}\right)} d x_{3} I\left(x_{i}\right) \\
& =\int_{2 \epsilon}^{1-2 \epsilon} d x_{2} \int_{0}^{\delta 2\left(1-x_{2}\right) x_{2}} d\left(1-x_{1}\right) I\left(x_{i}\right),
\end{aligned}
$$

apart from terms of order $\delta^{2}$, where

$$
I\left(x_{i}\right)=\left[\left(1-x_{1}\right)\left(1-x_{2}\right)\left(1-x_{3}\right)\right]^{D / 2} .
$$

The phase-space integration for the soft-momentum region $0 \leqslant x_{2} \leqslant 2 \epsilon$ is given by

$$
\int_{0}^{2 \epsilon} d x_{2} \int_{1-x_{3}}^{1} d x_{3} I\left(x_{i}\right)=\int_{0}^{2 \epsilon} d x_{2} \int_{0}^{x_{2}} d\left(1-x_{1}\right) I\left(x_{i}\right) \text {. }
$$

The actual calculation is simplified by making a suitable change of variables. The result is the inclusive cross section for the real-particle-emission processes

$\sigma_{1}(\epsilon, \delta)=\sigma_{0} \frac{\alpha_{C}}{\pi} f(D)\left\{C_{2}(G)\left[\left(\frac{4}{D^{2}}-\frac{11}{3} \frac{1}{D}\right) E^{D}-\left(4 \ln (2 \epsilon)+\frac{11}{3}\right) \ln \delta-\frac{5}{6} \pi^{2}+\frac{67}{9}\right]+N_{f} T(R)\left(\frac{4}{3} \frac{1}{D} E^{D}+\frac{4}{3} \ln \delta-\frac{23}{9}\right)\right\}$,

where finite terms proportional to $\epsilon$ and/or $\delta$ have been neglected. This expression agrees with our previous result, ${ }^{2}$ which was obtained by a different method of calculation. The Born diagram [Fig. 2(a)] and the virtual corrections (Fig. 4) lead to two back-to-back jets. They must be added to the cross section (3.5) to give the inclusive jet cross section to order $\alpha_{C}$,

$$
\sigma(\epsilon, \delta)=\sigma_{0}+\sigma(2)+\sigma_{1}(\epsilon, \delta)
$$

which is free of infrared divergences and mass singularities. Let us define the fraction $f(\epsilon, \delta)$ of all jetlike events which have a fraction $(1-\epsilon)$ of the total energy $E$ inside some pair of opposite cones of half angle $\delta$ :

$$
\begin{aligned}
f(\text { gluon }) & =\sigma(\epsilon, \delta) / \sigma_{\text {tot }} \\
& =1-\frac{\alpha_{C}}{\pi}\left\{\left[4 C_{2}(G) \ln (2 \epsilon)+\frac{11}{3} C_{2}(G)-\frac{4}{3} N_{f} T(R)\right] \ln (\delta)+\left(\frac{\pi^{2}}{3}-\frac{49}{36}\right) C_{2}(G)+\frac{2}{9} N_{f} T(R)\right\} .
\end{aligned}
$$

Note that the source-dependent $\ln (E / \mu)$ terms in $\sigma(\epsilon, \delta)$ and $\sigma_{\text {tot }}$ are canceled in the ratio $f(\epsilon, \delta)$. As noted earlier, the virtual correction $\sigma(2)$ has some ambiguity associated with the renor malization of the source. Owing to the multiplicative nature of the renormalization of the source, this ambiguity is common to $\sigma(\epsilon, \delta)$ and $\sigma_{\text {tot }}$ and therefore it disappears from the ratio $f(\epsilon, \delta)=\sigma(\epsilon, \delta) / \sigma_{\text {tot }}$. This ratio $f$, including the constant term in it, is independent of the renormalization of the source.
It is interesting to observe that the fraction $f(\epsilon, \delta)$ can be expressed directly in terms of the Altarelli-Parisi ${ }^{10}$ parton-splitting functions $P_{G G}(Z), P_{q G}(Z), P_{q q}(Z)$, and $P_{G q}(Z)$ (listed in the Appendix):

$f($ gluon $)=1-\frac{\alpha_{C}}{\pi}(\ln \delta) \int_{2 \epsilon}^{1-2 \epsilon} d Z\left[P_{G G}(Z)+P_{q G}(Z)\right]$, 
$f($ quark $)=1-\frac{\alpha_{C}}{\pi}(\ln \delta) \int_{2 \epsilon}^{1-2 \epsilon} d Z\left[P_{G_{q}}(Z)+P_{a q}(Z)\right]$,

where only logarithmic terms are kept. The fractions $f$ in Eqs. (3.7) and (1.1) (apart from the constant terms) are reproduced upon integrations over $Z$. In the Appendix we show the derivation of Eq. (3.8) in detail. It is clear from this analysis that the "jettiness" of quark jets and gluon jets is source independent.

Before the comparison of the quark jet and the gluon jet, let us consider the meaning of the coupling constant $\alpha_{C}$ in (3.7) and (3.9). These two expressions are based on one-loop calculations and $\alpha_{C}$ is a constant at this level. As suggested in Refs. 4 and 5, with suitable assumptions, these one-loop results can be generalized to the leadinglogarithmic approximation; namely, the coupling constant $\alpha_{C}$ can be replaced by the running coupling constant ${ }^{3} \alpha_{C}(E)$ which approaches zero as $E \rightarrow \infty$. Such a replacement is valid in the case of deepinelastic scattering where the short-distance effect and the long-distance effect are factorized by use of the operator-product expansion. Now let us look at the three-jet-like configuration in Fig. 2(1). If $\overrightarrow{\mathrm{p}}_{2}$ and $\overrightarrow{\mathrm{p}}_{3}$ are not soft (i.e., $x_{2} \geqslant 2 \epsilon$ and $x_{3}$ $\geqslant 2 \epsilon$ ) and $\theta \geqslant 2 \delta$, all momentum invariants such as $\overrightarrow{\mathrm{p}}_{1}^{2}, \overrightarrow{\mathrm{p}}_{2}^{2}=\left(\frac{1}{2} E x_{2}\right)^{2} z \epsilon^{2} E^{2}$ and $\left(\overrightarrow{\mathrm{p}}_{2}-\overrightarrow{\mathrm{p}}_{3}\right)^{2} \geqslant\left(\frac{1}{2} E\right)^{2} 2 x_{1} x_{2}$ $(1-\cos \theta) \approx 2 \epsilon \delta^{2} E^{2}$ increase with the total energy $E$. Such a reaction will be regarded as a shortdistance phenomenon and will be characterized by the QCD running coupling constant $\alpha_{C}(E)$ which decreases like $(\ln E)^{-1}$ as $E \rightarrow \infty$. On the other hand, if, e.g., $0 \leqslant x_{2} \leqslant 2 \epsilon$ or $0 \leqslant \theta \leqslant 2 \delta$ so that some momentum variables are no longer hard, then perturbative QCD cannot be applied to such configurations. However, for the inclusive cross section $\sigma(\epsilon, \delta)$ or the ratio $f(\epsilon, \delta)$ the contributions from these latter configurations are smeared over; the real-particle-emission contribution and the virtual correction add up to remove these longdistance singularities. Therefore, if $\epsilon$ and $\delta$ are chosen so that the (nonperturbative) long-distance effects are smeared over, the ratio $f(\epsilon, \delta)$ will represent the short-distance features of jet phenomena. For short-distance phenomena one can use an argument based on the renormalization group. As noted by Sterman and Weinberg, ${ }^{5}$ if the ratio $f=f\left(E / \mu, \alpha_{C} ; \epsilon, \delta\right)$ is free from infrared and mass singularities, it is rewritten as

$$
f\left(E / \mu, \alpha_{C} ; \epsilon, \delta\right)=f\left(1, \alpha_{C}(E) ; \epsilon, \delta\right),
$$

where $\alpha_{C}(E)$ is the coupling constant defined at the momentum of order $E$. With $\alpha_{C}$ replaced by
$\alpha_{C}(E)$, the perturbative results (3.7) and (1.1) are expected to be reliable at high energies; so is the total cross section (2.5).

In Fig. 5 we compare the quark-jet angular radius $\delta$ (quark) and the gluon-jet angular radius $\delta$ (gluon) for the same values of $f, \epsilon$, and $\alpha_{C}(E)$. To a good approximation

$\delta($ gluon $) \simeq[\delta \text { (quark) }]^{C_{2}(R) / C_{2}(G)}=[\delta(\text { quark })]^{4 / 9},(3.11)$

as noted earlier. ${ }^{2,6}$ The short-distance effects of QCD tend to make the gluon jet much broader than the quark jet. The energy dependence of the coupling constant $\alpha_{C}(E)$ tells us that the quark and gluon jets get narrower with increasing $E$. The narrowing of gluon jets, however, is slower than that of quark jets. This general qualitative feature is mainly a consequence of the difference in the color factors $C_{2}(R)$ and $C_{2}(G)$ associated with the representations of the quark and the gluon in QCD. (The larger the representation, i.e., the gross color charge, the larger the spreading of a jet.) The threshold energy for the detection of a clean gluon jet will be larger than that $(\sim 3 \mathrm{GeV})$ for the detection of a quark jet. This renders the detection of gluon jets very difficult. It is intriguing to consider this as the reason gluon jets have so far escaped detection.

The perturbative results (3.7) and (1.1), from which we can extract some qualitative features of jet phenomena at high energies, may not be adequate for quantitative analysis at present energies. This is because nonperturbative effects (fragmentation of quarks into hadrons) observed

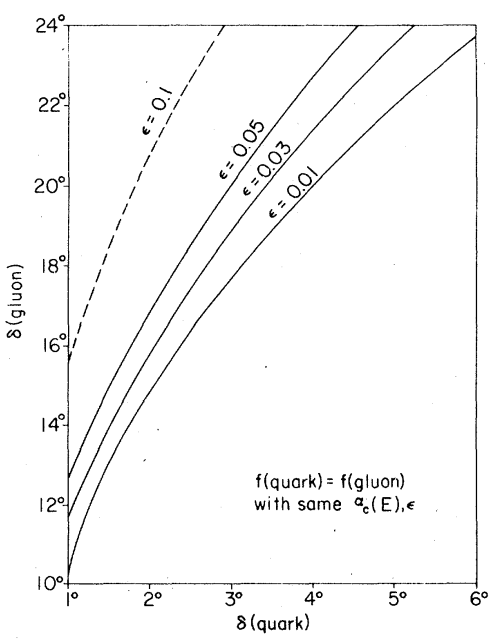

FIG. 5. Comparison of the gluon-jet angular radius and the quark-jet angular radius for fixed $f, \epsilon, \alpha_{C}$, $N_{f}=4$. The solid lines are valid only at very high energies. 
experimentally seem at least as important ${ }^{8}$ as the perturbative effects at the center-of-mass energy $E \sim 9 \mathrm{GeV}$ available at present. There is no inkling as to how a gluon fragments into hadrons. We do not know how large an angular radius $\delta$ is required to smear out the nonperturbative effects in a gluon jet. It is even possible that an energetic gluon fragments with such a large average transverse momentum that the jet formation never materializes. If this is the case, any QCD perturbative study of the gluon-jet structure is useless and we have to look for other means to test the existence of the gluon. For a rough estimate, let us suppose that the gluon fragmentation has a comparable limited transverse momentum as the quark fragmentation. Even in this case, $f(\epsilon, \delta)$ may not be a useful quantitative formula for energies available now or in the near future. Let us take the average transverse momentum to be $\left\langle p_{\perp}\right\rangle \sim 0.3 \mathrm{GeV} / c$. The nonperturbative effect on $\epsilon$ and $\delta$ will be $\epsilon_{\mathrm{NP}} \sim\left\langle p_{\perp}\right\rangle / E$ and $\delta_{\mathrm{NP}} \sim\left(\left\langle p_{\perp}\right\rangle\langle n\rangle\right) / E$, where $\langle n\rangle$ is the average hadron multiplicity. Typically, for $E=10 \mathrm{GeV}$ and $\langle n\rangle \sim 10$, we have $\epsilon_{\mathrm{NP}}(\sim 0.03)$ reasonably small but $\delta_{\mathrm{NP}}(\sim 0.3)$ uncomfortably large.

For a given energy, the perturbative effects are expected to dominate over the nonperturbative effects at large transverse momentum with respect to the jet axis. If we have the differential cross section expressed as a function of $p_{\perp}$, we can compare the quark- and the gluon-jet processes at large $p_{\perp}$ where nonperturbative effects are small. Following the suggestion of Farhi, ${ }^{8}$ let us use the thrust $T$ distribution [see Eq. (1.2)] to make a quantitative comparison of the quark-jet and gluon-jet productions.

For two-body configurations the thrust $T$ is proportional to $\delta(1-T)$. Therefore the Born cross section $\sigma_{0}$ and the virtual correction $\sigma(2)$ lead to the differential thrust cross sections $d \sigma_{0} / d T$ $=\sigma_{0} \delta(1-T)$ and $d \sigma(2) / d T=\sigma(2) \delta(1-T)$, respectively.

For three-body configurations the thrust is given by $T=\max \left(x_{1}, x_{2}, x_{3}\right)$ and $\frac{2}{3} \leqslant T \leqslant 1$. Let us suppose $T=x_{1}>x_{2}, x_{3}$ and integrate the differential cross section (2.1) over $x_{2}$ with $T=x_{1}$ kept fixed, taking into account the symmetry of the phase space. We obtain the differential cross section for the three-particle emission expressed in terms of thrust:

$$
\begin{array}{rl}
\frac{d \sigma(1)}{d T}=3 \int_{2(1-T)}^{T} & d x_{2}\left[\left(1-x_{1}\right)\left(1-x_{2}\right)\left(1-x_{3}\right)\right]^{D / 2} \\
& \times \frac{d^{2} \sigma(1)}{d x_{1} d x_{2}}
\end{array}
$$

where $T+x_{2}+x_{3}=2$. The factor $\left[\left(1-x_{1}\right)\left(1-x_{2}\right)\right.$ $\left.\left(1-x_{3}\right)\right]^{D / 2}$ serves to regularize the infrared divergences and mass singularities that occur at $T=1$, and can be set equal to one for $T \neq 1$. Therefore, except for $T=1$,

$$
\begin{aligned}
\frac{1}{\sigma_{0}} \frac{d \sigma(1)}{d T}=\frac{\alpha_{C}}{\pi}\left\{2\left[C_{2}(G)\left(T(1-T)+\frac{1}{T(1-T)}-2\right)+N_{f} T(R)(1-2 T(1-T))\right] \ln \left(\frac{2 T-1}{1-T}\right)\right. \\
\left.+(3 T-2)\left[C_{2}(G)\left(\frac{3}{2}+\frac{5}{2} T-\frac{11}{6} \frac{1}{1-T}\right)+N_{f} T(R)\left(-5 T+\frac{2}{3} \frac{1}{1-T}\right)\right]\right\} .
\end{aligned}
$$

It is evident that the leading singularities of $d \sigma(1) / d T$ at $T=1$ are canceled by those of the virtual correction $d \sigma(2) / d T=\sigma(2) \delta(1-T)$. [This is simply because the infrared divergences and mass singularities are canceled between $\sigma(1)$ and $\sigma(2)$.] In general, if we consider quantities such as $(1-T)^{n}(d \sigma / d T)(n \geqslant 1)$, it is not necessary to take explicit account of genuine two-body configurations (i.e., the Born term and the virtual correction) whose contributions are proportional to $\delta(1-T)$. Correspondingly, we shall henceforth consider the $(1-T)$ weighted, normalized thrust distribution $(1-T)^{n}\left(1 / \sigma_{\text {tot }}\right)(d \sigma / d T)(n \geqslant 1)$. Up to order $\alpha_{C}(E)$, we can write $(1-T)^{n}\left(1 / \sigma_{\text {tot }}\right)(d \sigma / d T)=(1-T)^{n}\left(1 / \sigma_{0}\right)[d \sigma(1) / d T]$, so that the renormalization-scheme dependence drops out trivially. In particular, the average of $(1-T)$ is given by

$$
\begin{aligned}
\langle 1-T\rangle^{\text {gluon }} & =\int_{2 / 3}^{1} d T(1-T)\left(1 / \sigma_{0}\right)(d \sigma / d T) \\
& =\frac{\alpha_{C}}{\pi}\left\{C_{2}(G)\left[\frac{11}{216}-\frac{43}{96} \ln 3-\frac{\pi^{2}}{6}+2 \ln 2 \ln 3+2\left[L\left(-\frac{1}{3}\right)-L\left(\frac{1}{3}\right)\right]\right]+N_{f} T(R)\left(-\frac{5}{108}+\frac{7}{48} \ln 3\right)\right\} \\
& =\frac{\alpha_{C}}{\pi}\left[C_{2}(G)(0.787)+N_{f} T(R)(0.114)\right] \\
& =\frac{\alpha_{C}}{\pi}(2.59)
\end{aligned}
$$


where we have included four flavors. $L(x)$ is the Spence function

$$
L(x)=\int_{0}^{x}(d y / y) \ln (1-y)
$$

We observe that most of the contribution to $\langle 1-T\rangle$ comes from the three-gluon vertex interaction. The spreading due to the quark-gluon interaction contributes roughly $10 \%$ to the total. It goes without saying that the spread of the gluon jet provides a very clean place for the measurement of the three-gluon vertex. For the quark jet, on the other hand, the differential cross section in terms of thrust is ${ }^{17}$

$$
\frac{1}{\sigma_{\text {tot }}} \frac{d \sigma \text { (quark) }}{d T}=\frac{\alpha_{C}}{\pi} C_{2}(R)\left[\left(\frac{2}{T(1-T)}-3\right) \ln \left(\frac{2 T-1}{1-T}\right)-\frac{3}{2}(3 T-2) \frac{2-T}{1-T}\right],
$$

and the averaged $1-T$ is

$$
\langle 1-T\rangle^{\text {quark }} \simeq \frac{\alpha_{C}}{\pi} C_{2}(R)(0.789) \simeq \frac{\alpha_{C}}{\pi}(1.05)
$$

In Fig. 6 we plot $(1-T)\left(1 / \sigma_{0}\right)(d \sigma / d T)$ for both the gluon case (solid line) and the quark case (dashed line). In addition, in Fig. 7 we show the ratio

$$
\left[\left(1 / \sigma_{0}\right)(d \sigma / d T)\right]_{\text {gluon }} /\left[\left(1 / \sigma_{0}\right)(d \sigma / d T)\right]_{\text {quark }},
$$

which turns out to be close to $C_{2}(G) / C_{2}(R)=\frac{9}{4}$ for the entire range $\frac{2}{3} \leqslant T<1 .^{18}$ This implies that a gluon jet and a quark jet will have, apart from the overall color charges, almost the same structure. Roughly speaking, for both types of jets, quantities which are related to the transversemomentum spread of a jet, such as $\langle 1-T\rangle$ and the spherocity, ${ }^{8}$ will be characterized by the ratio $C_{2}(G) / C_{2}(R)=\frac{9}{4}$. In particular, from (3.14) and (3.15) we learn that $\langle 1-T\rangle^{\text {gluon }} /\langle 1-T\rangle^{\text {quark }}=2.47$ $\sim C_{2}(G) / C_{2}(R)$; this is also true for the ratio of the "large- $T$ suppressed" $n$th moments $\left\langle(1-T)^{n}\right\rangle^{\text {gluon }} /$

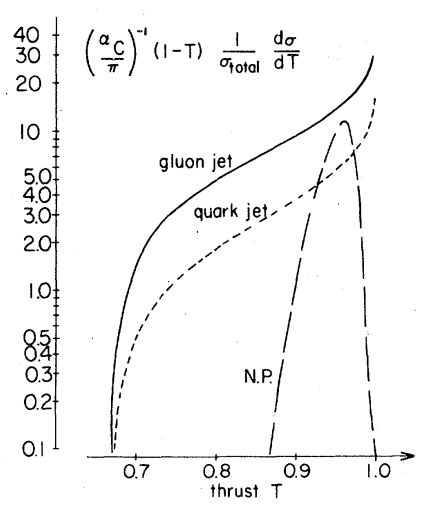

FIG. 6. Thrust distributions $\left(\alpha_{C} / \pi\right)^{-1}(1-T)\left(1 / \sigma_{\text {tot }}\right)$ $(d \sigma / d T)$. The solid line corresponds to the gluon jet while the dashed line corresponds to the quark jet. The long-dashed line corresponds to the thrust distribution for the quark fragmentation with exponential $p_{\perp}^{2}$ dependence at $E_{\mathrm{com}_{\mathrm{o}}}=16 \mathrm{GeV}$ with $\alpha_{C}(E=16 \mathrm{GeV}) \sim 0.21$. (See Ref. 17.)
$\left\langle(1-T)^{n}\right\rangle^{\text {quark }} \sim C_{2}(G) / C_{2}(R)(n \geqslant 1)$. The nonperturbative effect on $d \sigma / d T$ is important for large $T$ (see Fig. 6). To suppress this nonperturbative effect relative to the perturbative effect, it will be useful to consider the above-mentioned moments

$$
\left\langle(1-T)^{n}\right\rangle \equiv \int_{2 / 3}^{1} d T(1-T)^{n}\left(1 / \sigma_{\text {tot }}\right)(d \sigma / d T)(n \geqslant 1) .
$$

In Fig. 6, we have included the $(1-T)$ weighted thrust distribution for the quark fragmentation, which is obtained phenomenologically ${ }^{17}$ from experiment. In Fig. 8 , where the $(1-T)^{2}\left(1 / \sigma_{\text {tot }}\right)(d \sigma /$ $d T)$ distributions are plotted, we observe that the nonperturbative effects are suppressed for $T<0.9$, where the perturbative effects begin to dominate. The effects of higher-order perturbative QCD contributions can best be measured for $T$ values between $\frac{1}{2}$ and $\frac{2}{3}$.

\section{DISCUSSION AND REMARKS}

The hadronic decay of a quarkonium state via the three-gluon intermediate mode has been discussed exhaustively in the literature. ${ }^{19}$ Direct observation of gluon jets from $\Upsilon$ decay is anticipated. However, as a consequence of our analysis on the gluon jets, the following distinct possibility

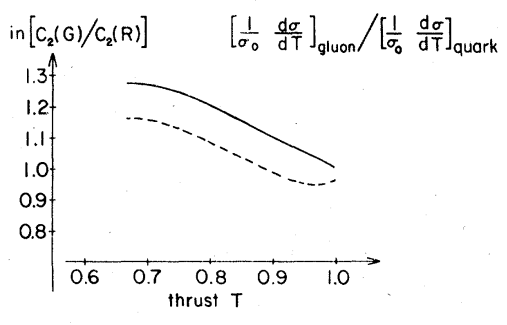

FIG. 7. The ratio of the differential cross sections $\left(1 / \sigma_{0}\right)(d \sigma / d T)$ for the gluon-jet and quark-jet cases. The vertical scale is measured in units of $C_{2}(G) / C_{2}(R)$ $=9$. The dashed line represents the contribution to $(d \sigma / d T)$ (gluon) from the gluon-gluon interaction only. The solid line includes the quark-gluon interaction as well $\left(N_{f}=4\right)$. 


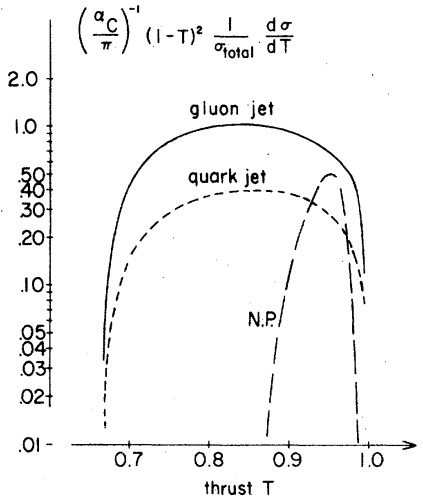

FIG. 8. The $n=2$ thrust distribution $\left(\alpha_{C} / \pi\right)^{-1}(1-T)^{2}$ $\left(1 / \sigma_{\text {tot }}\right)(d \sigma / d T)$. The solid and dashed lines correspond to the gluon and quark jets, respectively. The longdashed line corresponds to the quark fragmentation.

emerges: The gluon jets from $\Upsilon$ decay are not observable; that is, prohibitive statistics would be required to sort out the three-jet structure in the hadronic decays of the $\Upsilon$. If this is the case, we are left with few alternatives to search for the gluon-jet structure in the quarkonium decay. The best solution to this problem is provided by the discovery of a very heavy quark. The heavier the quark mass, the more energetic are the gluons produced from the decay. Since the gluon jet narrows as the energy of the gluon increases, the detection of the three-gluon-jet structure becomes more accessible. In this section we give a brief discussion on what we consider to be the best way to search for the gluonjet structure in $\Upsilon$ decay.

The property that is easiest to find in the analysis of jet structures is the jet axis. Even for a multijet event, we can still define the thrust axis by maximizing the momenta parallel to it. Once the thrust axis is determined for each hadronic decay event, we can then study its angular distribution with respect to a fixed direction, say, the $e^{+} e^{-}$beam direction. We call this angle $\theta_{T}$.

It is straightforward to derive the differential decay cross section for the three-gluon decay of an orthoquarkonium state ${ }^{20}$ :

$$
\frac{1}{\Gamma_{3 g}} \frac{d \Gamma_{3 g}}{d T d \cos \theta_{T}}=\frac{9}{\left(\pi^{2}-9\right)} \frac{1}{4 T^{2}(2-T)^{2}}\left[A(T)\left(1+\cos ^{2} \theta_{T}\right)-B(T)\left(1-3 \cos ^{2} \theta_{T}\right)\right],
$$

where

$$
\begin{aligned}
& A(T)=\frac{(3 T-2)\left(2-T^{2}\right)}{T}+\frac{2(1-T)\left(5 T^{2}-12 T+8\right)}{2-T} \ln \left(\frac{2-2 T}{T}\right) \\
& B(T)=\frac{1-T}{T^{2}}\left[\frac{(3 T-2)\left(2 T^{3}-7 T^{2}+6 T+2\right)}{T}+\frac{2\left(T^{4}-6 T^{3}+18 T^{2}-24 T+12\right)}{2-T} \ln \left(\frac{2-2 T}{T}\right)\right]
\end{aligned}
$$

and $\Gamma_{3 g}$ is the three-gluon decay width of a heavy quarkonium state [see Fig. 9(a)]. We note that as $T \rightarrow 1, B(T) \rightarrow 0$ and

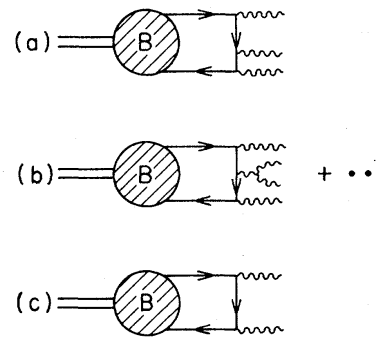

FIG. 9. (a) The three-gluon decay mode of an orthoquarkonium state; (b) a typical higher-order diagram for the decay of a quarkonium state; (c) the two-gluon decay mode of a paraquarkonium or a $P$ state.

$$
\frac{1}{\Gamma_{3 g}} \frac{d \Gamma_{3 g}}{d T d \cos \theta_{T}} \rightarrow \frac{9}{\left(\pi^{2}-9\right)} \frac{1}{4}\left(1+\cos ^{2} \theta_{T}\right) .
$$

This is the same angular distribution as the background $e^{+} e^{-}-\gamma^{*} \rightarrow \bar{q} q$ and the hadronic decay via a virtual photon. For values of $T$ away from unity, the angular dependence and the differential cross section both decrease, as shown clearly in Fig. 10. For example, at $T=0.8$,

$$
\left.\frac{1}{\Gamma_{3 g}} \frac{d \Gamma_{3 g}}{d T d \cos \theta_{T}}\right|_{T=0.8} \simeq \frac{9}{\pi^{2}-9} \frac{1}{9}\left(1+0.2 \cos ^{2} \theta_{T}\right) .
$$

The angular dependence should provide a better test of the three-gluon decay mode than a single $T$-dependent differential cross section. To see this, let us integrate over the angle $\theta_{T}$ to obtain ${ }^{17}$

$$
\frac{1}{\Gamma_{3 g}} \frac{d \Gamma_{3 g}}{d T}=\frac{6}{\left(\pi^{2}-9\right)} \frac{A(T)}{T^{2}(2-T)^{2}} .
$$




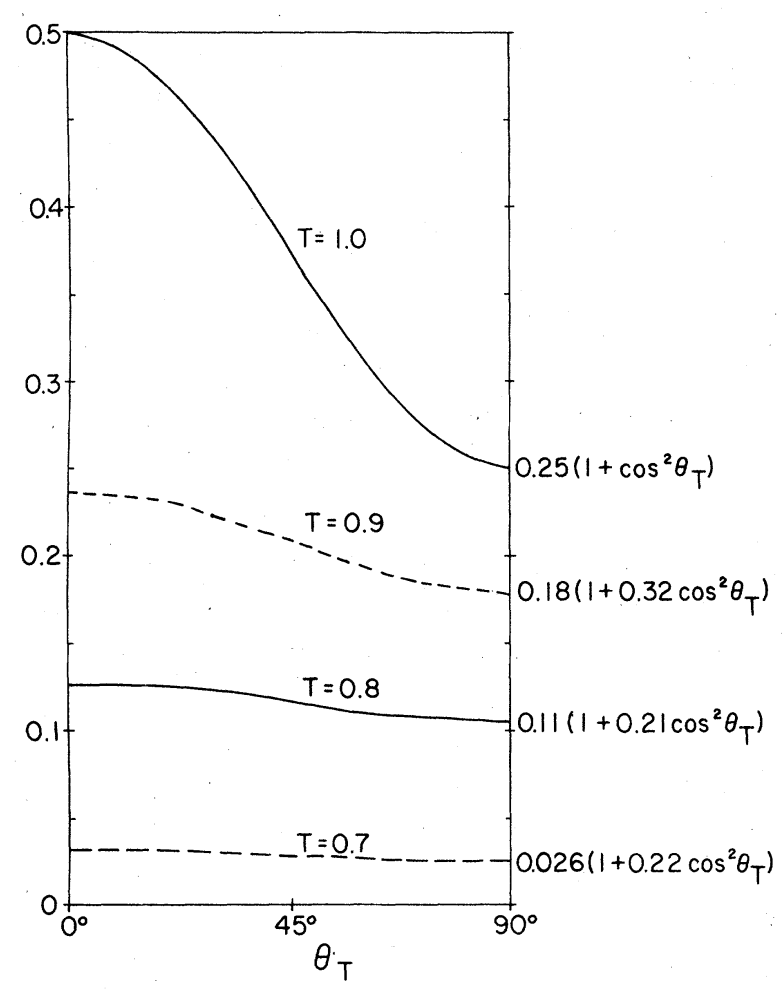

FIG. 10. The plot of

$$
\frac{1}{\Gamma_{3 g}}\left(\frac{\pi^{2}-9}{9}\right) \frac{d \Gamma_{3 g}}{d \cos \theta_{T} d T}
$$

as a function of the angle $\theta_{T}$ for different values of $T$.

This $T$ distribution is plotted in Fig. 11, where we have also plotted a pure three-body phasespace distribution (properly normalized):

$$
\left(1 / \sigma_{\text {tot }}\right)(d \sigma / d T)=6(3 T-2) \text {. }
$$

The two distributions (4.4) and (4.5) look almost identical, with the three-gluon decay distribution slightly more peaked at $T=1$. In fact they have the same average thrust

$$
\begin{aligned}
\langle 1-T\rangle_{3 g} & =\langle 1-T\rangle_{3-\text { body phase space }} \\
& =0.111 .
\end{aligned}
$$

This means that the thrust distribution of the $\Upsilon$ decay does not provide a sensitive test of the vector nature of the gluons. This is in contrast with the bremsstrahlung processes, where the amplitudes have enhancements due to infrared and mass singularities, giving rise to drastic deviations from the phase space.

Here we have tacitly assumed that the angular

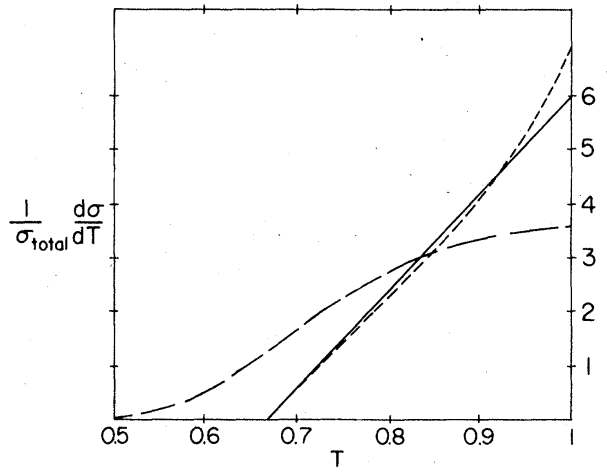

FIG. 11. The thrust distribution of $\left(1 / \sigma_{\text {tot }}\right)(d \sigma / d T)$. The solid line is for the three-body phase space. The dashed line is for the three-gluon decay. The longdashed line is for the four-body toy model. The last is provided to illustrate a typical four-body thrust distribution.

distribution (Fig. 10) calculated from the lowestorder three-gluon decay diagram is not drastically altered by higher-order corrections. These corrections include (1) higher-order perturbative QCD corrections [such as shown in Fig. 9(b)] as well as (2) nonperturbative effects, e.g., the internal motion of quarks inside the quarkonium. Careful evaluation of these corrections (which may be sizable) is beyond the scope of this paper. Phenomenologically, we can learn something about the size of the corrections by looking at the $T$ distribution. A pure three-body decay [such as Fig. 9 (a)] covers the range $\frac{2}{3} \leqslant T \leqslant 1$. Owing to the higher-order corrections, a quarkonium state can decay via four (or more) gluons (and/or light quarks) into hadrons; then the thrust distribution becomes nonzero for values of thrust down to $T \geqslant \frac{1}{2}$. A measurement of such events with $\frac{1}{2}$ $\leqslant T \leqslant \frac{2}{3}$ should give us some indication of the size of the higher-order corrections. In Fig. 11, we have, for the purpose of illustration, also plotted the thrust distribution of a four-body final-state toy model (for convenience, we choose the matrix element to be a smooth-behaving function such that the thrust axis is always along the direction of the most energetic particle). It is properly normalized, with an average thrust $\langle 1-T\rangle_{4-\text { body }}$ $=0.16$; this larger value for $\langle 1-T\rangle$ will typically characterize the contribution of four- (or more-) body phase-space models.

We should point out that the experimental thrust distribution of a quarkonium decay into hadrons is expected to peak toward large $T$ more than that shown in Fig. 11. This is due to the electromagnetic decay mode $(Q \bar{Q}) \rightarrow \gamma^{*} \rightarrow q \bar{q} \rightarrow$ hadrons, where $Q$ is a heavy quark and $q$ is a light quark. Fortu- 
nately this decay mode can be determined accurately from the measurements of the background $e^{+} e^{-} \rightarrow \gamma^{*} \rightarrow q \bar{q} \rightarrow$ hadrons and the leptonic width of the $1^{--}$quarkonium state.

We conclude the discussion with a few remarks.

(1) We do not expect to observe the gluon-jet structure in $\Upsilon$ decay. Probably even the acoplanarity structure is not easy to observe. It is difficult to estimate the threshold energy of a gluon above which its jet structure is observable. Our best guess of this threshold value is somewhere above $5 \mathrm{GeV}$ per jet.

(2) The three-gluon decay mode of the quarkonium state $\left(1^{--}\right)$is infrared finite. ${ }^{19} \mathrm{~A}$ comparison of its thrust and sphericity distributions would reveal properties of the gluon fragmentation. Properties such as multiplicity and quantum-number correlations are of utmost interest, even though they cannot be unambiguously calculated within the framework of perturbative QCD. ${ }^{21}$

(3) Theoretically, the hadronic decay of a pseudoscalar (or a higher angular momentum) state via two gluons [Fig. 9(c)] would be most useful, ${ }^{22}$ since this decay width is much larger than that of the three-gluon decay mode. Typically we arrive at the decay of a state $X$ via radiative transition

$$
\left(1^{--}\right)-\gamma+X
$$

for any quarkonium system. We can always go to the rest frame of the state $X$ by measuring the photon momentum. Our Eqs. (3.7) and (3.13) should be directly applicable to the hadronic decay of $X$.

(4) As pointed out originally by Appelquist and Politzer, ${ }^{23}$ the hadronic decay of an orthoquarkonium state provides a measurement of the QCD coupling

$$
\begin{aligned}
R_{Q} & =\frac{\Gamma_{\text {had }}}{\Gamma_{\mu}+\mu-} e_{Q}{ }^{2}=C_{1}\left[\alpha_{C}(E)\right]^{3} \\
& =C_{2}\left(\ln \frac{E}{\Lambda}\right)^{-3}
\end{aligned}
$$

where $C_{1}, C_{2}$ are known constants and $e_{Q}$ is the fraction of the electric charge of the heavy quark $Q$. This cubic logarithmic dependence on the bound-state mass $E$ provides a good test of perturbative QCD. However, there may be parts of the phase space where some of the energy-momentum invariants involved are not large enough to justify the use of perturbative QCD. ${ }^{24}$ The situation can be somewhat improved by considering the partially integrated $\Gamma_{3 g}$, say $\Gamma_{3 g}\left(T_{0}\right)$,

$$
\Gamma_{3 g}\left(T_{0}\right)=\int_{1 / 2}^{T_{0}}\left(d \Gamma_{3 g} / d T\right) d T
$$

instead of $\Gamma_{3 g}\left[\Gamma_{3 g}\left(T_{0}=1\right)=\Gamma_{3 g}\right]$ since for $T$ away from 1 , all energy-momentum invariants involved are large. Hence we replace Eq. (4.7) by

$$
R_{Q}\left(T_{0}\right)=e_{Q}{ }^{2}\left[\Gamma_{\text {had }}\left(T_{0}\right) / \Gamma_{\mu}+\mu-\right] .
$$

If we take $\Lambda=500 \mathrm{MeV}$, we have

$$
\frac{R_{\Psi}}{R_{\Upsilon}}=\frac{R_{\Psi}\left(T_{0}\right)}{R_{\Upsilon}\left(T_{0}\right)} \sim 4
$$

for any value of the thrust threshold $T_{0} \neq 1$.

(5) Although $\sigma_{\text {tot }}$ of the scalar (or the pseudoscalar) source is different from the $\sigma_{\text {tot }}$ of the pseudoscalar quarkonium state decay into hadrons, they have the same $f(\epsilon, \delta)$ as given in Eq. (3.7). In fact, to the approximation we are considering, the spread in transverse momentum as a function of the total energy of a gluon jet is independent of how it is produced. Therefore, we expect the same broadness for the gluon jets produced in different processes. At sufficiently high energies, gluon jets are expected in deep-inelastic scattering, Drell-Yan dilepton production, large- $p_{\perp}$ scatterings, and $e^{*} e^{-}$annihilation into hadrons. We emphasize again that the transverse-momentum spread of a gluon jet provides a very clean measurement of the three-gluon vertex.

\section{ACKNOWLEDGMENT}

We thank C. K. Lee and our colleagues at Fermilab and Cornell, in particular W. A. Bardeen, for useful discussions. The work of S.-H.H.T. was supported in part by the National Science Foundation.

\section{APPENDIX}

In this appendix we shall outline the calculation of the inclusive jet cross section for the colorsinglet, gauge-invariant pseudoscalar source $F_{\mu \nu}^{a}[A] \tilde{F}_{\mu \nu}^{a}[A]=\frac{1}{2} \epsilon_{\mu \nu \rho \tau} F_{\mu \nu}^{a}[A] F_{\rho \tau}^{a}[A]$. In particular, we shall express the cross section for real-particle-emission processes in terms of the parton splitting functions of Altarelli and Parisi. ${ }^{10}$

The pseudoscalar source $F_{\mu \nu}^{a}[A] \tilde{F}_{\mu \nu}^{a}[A]$ effectively represents the two-gluon decay of a $0^{-+}$ quark-antiquark bound system in the limit of heavy quark mass. ${ }^{25}$ To discuss this source in the framework of dimensional regularization, special care must be taken to define the Levi-Civita tensor $\epsilon_{\mu \nu \rho \tau}$ (which is peculiar to four dimensions) in arbitrary dimensions. 'In four dimensions the product of two of these tensors is expressed in the form of a determinant

$$
\epsilon_{\mu_{1} \mu_{2} \mu_{3} \mu_{4}} \epsilon_{\nu_{1} \nu_{2} \nu_{3} \nu_{4}}=-\operatorname{det}\left(g_{\mu_{i} \nu_{j}}\right) \quad(i, j=1-4) .
$$

Since only such products appear in our present case, we shall define the $n(=4+D)$-dimensional 
generalization of this product by the right-hand side of Eq. (A1).

In the real-particle-emission processes shown in Fig. 2 a virtual gluon (with momentum $p$ ) decays into two gluons or ghosts or quarks (with momentum $k$ and $l$ ). Let us denote by $\theta$ the angle between the three-momenta $\vec{p}$ and $\vec{k}$ in the centerof-mass frame, and introduce the fractional energy $Z=2 k^{0} / E, 0 \leqslant Z \leqslant 1$. In terms of these variables the cross section is given by

$$
\sigma=\sigma_{0}\left(8 \pi^{2}\right)^{-1} f(D)\left(\frac{1}{2} E\right)^{2+D} \int d Z[Z(1-Z)]^{1+D} \int d \cos \theta\left[(\sin \theta)^{D} /\left(1-Z \cos ^{2} \frac{\theta}{2}\right)^{2+D}\right] \mathcal{F},
$$

where $\mathcal{F}$ is the square of the one-loop Feynman amplitude $\mathfrak{M}$ divided by the lowest-order amplitude $\mathfrak{M}_{0}$, $\mathfrak{F}=\mid \mathfrak{M}(1-$ loop $) /\left.\mathfrak{T}_{0}\right|^{2}$. In the Feynman gauge the contributions from diagrams Figs. 2(f)-2(j) are given by

$$
\begin{aligned}
& \mathcal{F}^{(\mathrm{f})+(\mathrm{g})+(\mathrm{h})+(\mathrm{i})}=2 g^{2}\left(\left\{C_{2}(G)[1+2 Z(1-Z)]+N_{f} T(R) 2[1-(2-D) Z(1-Z)]\right\}\left(1 / p^{2}\right)+\cdots\right), \\
& \mathcal{F}^{(\mathrm{j})}=2 g^{2} C_{2}(G)\left\{4[(2-Z)(1-Z) / Z(1-\cos \theta)]\left(1 / p^{2}\right)+\cdots\right\}
\end{aligned}
$$

where only terms singular for $p^{2}-0$ are retained. (The contributions from the mirror image diagrams have also been included.) As may be checked easily, the $1 / p^{2}$ terms in $\mathcal{F}$ take exactly the same form for both scalar and pseudoscalar sources (diagram by diagram). It is simple to see that the leading contribution (i.e., excluding nonleading terms of order $\epsilon$ and/or $\delta$ ) to the jet cross section comes from those parts of $\mathcal{F}$ that are singular for $p^{2} \rightarrow 0$. This, combined with the above fact, implies that both sources lead to the same short-distance features of gluon jets [i.e., Eqs. (3.7), (3.13), and (3.14)].

The hard-momentum region which gives rise to mass singularities is characterized by the phase space $2 \epsilon \leqslant Z \leqslant 1-2 \epsilon$ and $0 \leqslant \theta \leqslant 2(1-Z) \delta$, where the latter follows from the restriction that the angle between $\overrightarrow{\mathrm{k}}$ and $\vec{i}$ be less than $2 \delta$. Note that Fig. 2(b) involves two collinear configurations $\vec{k} \| \vec{i}$ or $(-\vec{k} \| \vec{p})$ that lead to mass singularities. The infrared divergences arise from the soft-momentum configuration $0 \leqslant Z \leqslant 2 \epsilon$ and $0 \leqslant \theta \leqslant \pi$ as well as another similar configuration corresponding to $0 \leqslant 1-Z \leqslant 2 \epsilon$. Let us add up and rearrange the contributions from the hard-and soft-momentum configurations within the phase-space integral for each amplitude in (A3). Then we perform the angular integration and put all the expressions together. The result is the cross section

$$
\begin{aligned}
\sigma_{1}=\sigma_{0}\left(\alpha_{C} / \pi\right) f(D) & {\left[\left(\frac{1}{D}-\frac{1}{24} \pi^{2} D\right) \int_{0}^{1} d Z[2 Z(1-Z)]^{D}\left[P_{G G}(Z)+P_{q G}(Z)\right]\right.} \\
& \left.+(\ln \delta) \int_{2 \epsilon}^{1-2 \epsilon} d Z\left[P_{G G}(Z)+P_{q G}(Z)\right]+\frac{1}{3} N_{f} T(R)\right]
\end{aligned}
$$

where $P_{G G}(Z)$ and $P_{q g}(Z)$ are the parton-splitting functions ${ }^{1_{0}}$

$$
\begin{aligned}
& P_{G G}(Z)=C_{2}(G)\left[\frac{1-Z}{2}+\frac{Z}{1-Z}+Z(1-Z)\right], \\
& P_{q G}(Z)=N_{f} T(R)\left[Z^{2}+(1-Z)^{2}\right] .
\end{aligned}
$$

In the above expression (A4), the limit $D \rightarrow 0$ is understood and terms of order $\epsilon$ and/or $\delta$ are neglected. The $1 / D$ term in (A4) represents mass singularities arising from the angular integration, e.g.,

$$
\begin{aligned}
\int_{0}^{\pi} d \theta(\sin \theta)^{D / 2} & =B\left(\frac{1}{2}, \frac{1}{2} D\right) \\
& =\left(2^{1+D} / D\right)\left[1-\frac{1}{24} \pi^{2} D^{2}+O\left(D^{3}\right)\right]
\end{aligned}
$$

The constant term $\frac{1}{3} N_{f} T(R)$ in (A4) is related to the use of dimensional regularization; in fact, we could remove it by assigning suitable $D=n-4$ depen- dence to the trace $\operatorname{Tr}\left(\gamma_{\mu} \gamma_{\nu}\right)$ (which is chosen to be $4 g_{\mu \nu}$ in our present calculation) so that $\operatorname{Tr}\left(\gamma_{\mu} \gamma_{\nu}\right)$ $=(4-D) g_{\mu \nu}$. Of course, all physical results are independent of the choice of definition, as long as it is used consistently. The integration over $Z$ leads to our previous result (3.5) and hence the fraction $f(\epsilon, \delta)$ in Eq. (3.7).

The interpretation of (A4) in terms of the generalized parton language is obvious. The parton language, in particular, suggests that the structure of the lno term in (A4) is quite universal, being independent of how gluon and quark jets are produced.

It is straightforward to calculate the fraction $f(\epsilon, \delta)$ for the quark-jet case. ${ }^{26}$ Equation (3.9) is obtained after the cancellation of divergences. The parton functions ${ }^{1_{0}}$ are

$$
\begin{aligned}
& P_{\alpha q}(Z)=C_{2}(R)\left(1+Z^{2}\right) /(1-Z), \\
& P_{G Q}(Z)=C_{2}(R)\left[1+(1-Z)^{2}\right] / Z .
\end{aligned}
$$


*Operated by Universities Research Association, Inc. under contract with the Energy Research and Development Administration.

${ }^{1} \mathrm{G}$. Hanson et al., Phys. Rev. Lett. 35, 1609 (1975); G. Hanson, in Proceedings of the $\overline{X V I I I ~ I n t e r n a t i o n a l ~}$ Conference on High Energy Physics, Tbilisi, 1976, edited by N. N. Bogolubov et al. (JINR, Dubna, U.S.S.R., 1977), Vol. II, p. 81; PLUTO Collaboration, DESY report, 1978 (unpublished); DASP Collaboration, DESY report, 1978 (unpublished).

${ }^{2}$ K. Shizuya and S.-H. H. Tye, Phys. Rev. Lett. 41 , 787 (1978); 41, 1195(E) (1978).

${ }^{3}$ H. D. Politzer, Phys. Rev. Lett. 30, 1346 (1973); D. J. Gross and F. A. Wilczek, ibid. 30, 1943 (1973); Phys. Rev. D 9 , 980 (1974); H. Georgi and H. D. Politzer, ibid. $\underline{9}, 416$ (1974). See also N. Christ, B. Hasslacher, and A. H. Mueller, ibid. $\underline{6}$, 3545 (1972).

${ }^{4}$ H. D. Politzer, Phys. Lett. 70B, 430 (1977); Nucl. Phys. B129, 301 (1977). For more recent advances, see D. Amati, R. Petronzio, and G. Veneziano, ibid. B140, 54 (1978); B146, 29 (1978); R. K. Ellis, H. Georgi, M. Machacek, H. D. Politzer, and G. G. Ross, Nucl. Phys. B152, 285 (1979); A. H. Mueller, Phys. Rev. D 18, 3705 (1978). See also V. N. Gribov and L. N. Lipatov, Yad. Fiz. 15, 1218 (1972) [Sov. J. Nucl. Phys. 15, 675 (1972)]; Yu. L. Dokshitser, D. I. D'yakonov, and S. I. Troyan, in Proceedings of the 13th Leningrad Winter School, 1978 (unpublished) [English translation: SLAC Report No. SLAC TRANS183 (unpublished)]; C. H. Llewellyn Smith, lectures at the XVII Internationale Universtätswochen für Kernphysik, Schladming, Austria, Oxford Report No.47/78, 1978 (unpublished).

${ }^{5}$ G. Sterman and S. Weinberg, Phys. Rev. Lett. 39, 1436 (1977).

${ }^{6}$ M. B. Einhorn and B. Weeks, Nucl. Phys. B146, 445 (1978).

${ }^{7}$ A. V. Smilga and M. I. Vysotsky, Report No. ITEP-93, 1978 (unpublished). These authors give an extensive study of infrared divergences and mass singularities at the one-loop level in QCD.

${ }^{8} \mathrm{E}$. Farhi, Phys. Rev. Lett. 39, 1587 (1977). See also H. Georgi and M. Machacek, ibid. 39, 1237 (1977); A. De Rújula, J. Ellis, E. G. Floratos, and M. K. Gaillard, Nucl. Phys. B138, 387 (1978).

${ }^{9}$ T. Kinoshita, J. Math. Phys. 3 , 650 (1962); T. D. Lee and M. Nauenberg, Phys. Rev. 133, B1549 (1964). Recently, the problem of mass singularities and infrared divergences has been discussed extensively in the literature. See, for example, J. Frenkel and J. C. Taylor, Nucl. Phys. B116, 185 (1976); T. Kinoshita and A. Ukawa, Phys. Rev. D 16, 332 (1977); A. Sugamoto, ibid. 16, 1065 (1977); S. Libby and G. Sterman, ibid. 18, 3252 (1978); 18, 4737 (1978); Stony Brook Report No. SB-41, 1978 (unpublished); Ref. 4. Other earlier works can be found in the above references.

${ }^{10}$ G. Altarelli and G. Parisi, Nucl. Phys. B126, 298 (1977).

${ }^{11}$ The idea of smearing over nonperturbative effects is first emphasized by E. Poggio, H. Quinn, and S.
Weinberg, Phys. Rev. D 13, 1958 (1976). See also, R. Shankar, ibid. 15, 755 (1977).

${ }^{12} \mathrm{G}$. 't Hooft and M. Veltman, Nucl. Phys. B44, 189 (1972); C. G. Bollini and J. J. Giambiagi, Nuovo Cimento 12B, 120 (1972); W. J. Marciano and A. Sirlin, Nucl. Phys. B88, 86 (1975); R. Gastmans and R. Mueldermans, ibid. B63, 277 (1973).

${ }^{13}$ The Lorentz-invariant three-body phase space in $n$ dimensions

$$
d \Gamma=\delta^{(n)}\left(Q-\sum_{i=1}^{3} p_{i}\right) \prod_{i=1}^{3} d^{n-1} p_{i} / 2 E_{i},
$$

where $Q_{\mu}=(E, \overrightarrow{0})$ is the center-of-mass total momentum, is related to the phase space $d R$ expressed in terms of $x_{i}$ [Eq. (2.2)] so that

$$
d \Gamma=\frac{1}{8} B\left(\frac{1}{2}, 1+\frac{1}{2} D\right) C_{1}{ }^{2}\left(\frac{1}{2} E\right)^{2+D} d R,
$$

where $C_{1}=2 \pi^{1+D / 2} / \Gamma\left(1+\frac{1}{2} D\right)$ and $D=n-4$.

${ }^{14}$ See, e.g. , H. Kluberg-Stern and J. B. Zuber, Phys. Rev. D 12, 467 (1975); C. K. Lee, ibid. 14, 1078 (1976); S. D. Joglekar and B. W. Lee, Ann. Phys. (N. Y.) 97, 160 (1976).

${ }^{15} \mathrm{C}$. Q. Weizsäcker and E. J. Williams, Z. Phys. 88 , 612 (1934).

${ }^{16}$ The method of evaluating $f(\epsilon, \delta)$ used here is close to that used by P. M. Stevenson, Phys. Lett. 78B, 451 (1978); Einhorn and Weeks, Ref. 6. Here we take great care to demonstrate the cancellation of divergences and the evaluation of the constant term in $f(\epsilon, \delta)$.

${ }^{17}$ De Rújula et al. , Ref. 8.

${ }^{18} \mathrm{~K}$. Konishi, A. Ukawa, and G. Veneziano, Phys. Lett. 80B, 259 (1979); R. K. Ellis and R. Petronzio, ibid. $\overline{80 \mathrm{~B}}, 249$ (1979), where related analyses have been carried out.

${ }^{19} \mathrm{~T}$. Appelquist and H. D. Politzer, Phys. Rev. Lett. 34, 43 (1975); Phys. Rev. D 12, 1404 (1975); T. A. DeGrand, Y. J: Ng, and S. -H. H. Tye, ibid. 16, 3251 (1977). A list of more recent references can be found in Ref. 17.

${ }^{20}$ We agree with $\mathrm{K}$. Koller and T. F. Walsh, Nucl. Phys. B140, 449 (1978), except for an overall normalization factor.

${ }^{21}$ For a review of the application of QCD to the study of jet structure which goes beyond the framework of perturbative QCD, see, e.g., S. J. Brodsky, SLAC Report No. SLAC-PUB-2217, 1978 (unpublished).

${ }^{22}$ See, e.g. , H. Fritzsch and K. -H. Streng, Phys. Lett. 74B, 90 (1978); S. J. Brodsky, D. G. Coyne, T. A. DeGrand, and R. R. Horgan, ibid. 73B, 203 (1978); M. Krammer and H. Kraseman, ibid. 73B, 58 (1978). ${ }^{23}$ T. Appelquist and H. D. Politzer, Ref. 19 .

${ }^{24}$ Reference 23 . See also our discussion in Sec. III.

${ }^{25}$ See, e.g., J. M. Jauch and F. Rohrlich, The Theory of Photons and Electrons (Addison-Wesley, Reading, Mass., 1955), Chap. 12. The two-photon decay of the $1^{1} S_{0}$ state of positronium has the same structure as the decay from the pseudoscalar source $F_{\mu \nu} \tilde{F}_{\mu \nu}$.

${ }^{26} \mathrm{We}$ learned that a comparable analysis on the quark-jet structure has been carried out by W. Furmanski, Cracow Report No. TPJU 11/78 (unpublished). 